\title{
Les couleurs du ciel
}

\section{Camille Risi', Venance Journé', Jean-Louis Dufresne', Jean-Yves Grandpeix', Philippe Dubuisson ${ }^{2}$, Martin Turbet ${ }^{1}$}

1 Laboratoire de météorologie dynamique, Institut Pierre-Simon Laplace, Université Pierre-et-Marie-Curie / CNRS, Paris

2 Laboratoire d'optique atmosphérique, Université de Lille sciences et technologies, Villeneuve d'Ascq

camille.risi@Imd.jussieu.fr venance.journe@lmd.jussieu.fr

But pédagogique : comprendre les différentes couleurs que l'on voit dans le ciel et les nuages.

Public : du CE2 au lycée : discours adaptable.

Matériel nécessaire : trois aquariums transparents, incolores, très propres et identiques (en verre ou en plastique) de taille minimale de 10 sur $20 \mathrm{~cm}$, un éclairage avec de la lumière blanche et un faisceau suffisamment focalisé (par exemple, un projecteur de diapositives), de l'eau, un peu de lait (liquide ou en poudre, dans les deux cas, il faudra s'assurer que le lait est suffisamment frais), un peu de talc, de quoi mélanger (deux cuillères ou baguettes), un récipient (bol ou bouteille), une feuille de papier blanc et/ou un écran blanc, une feuille de papier noir et/ou un écran noir. L'expérience doit être effectuée dans une pièce sombre.

\section{Sites internet :}

www.ipsl.fr/fr/Pour-tous/Espacepedagogique/

www.lmd.jussieu.fr/ jldufres/Manip/ couleur_ciel.html
$\mathbf{P}$ ourquoi le ciel est-il bleu ? Nous y sommes tellement habitués que cette question en est déroutante. «C'est le bleu de la mer qui se reflète dans le ciel... » avait un jour proposé une petite fille pleine de poésie. Cet article propose une série d'expériences analogiques permettant de comprendre les raisons pour lesquelles le ciel est bleu, devient orange, voire rouge au lever et au coucher de soleil, et pour lesquelles les nuages sont blancs ou gris (Dufresne, 2002). Pédagogiquement, il est préférable de commencer par l'expérience montrant qu'un ciel nuageux est blanc ou gris, et seulement ensuite d'en venir au bleu du ciel. L'exploitation pédagogique peut facilement être adaptée en fonction du public, des écoliers du primaire aux lycéens, et elle convient au grand public lors de manifestations d'éducation scientifique. Nous présentons ici une série de quatre expériences pour appuyer la discussion sur les différentes couleurs du ciel. Les encadrés sur la lumière, l'absorption, la diffusion et les diffusions de Rayleigh et de Mie rappellent les principes de base.
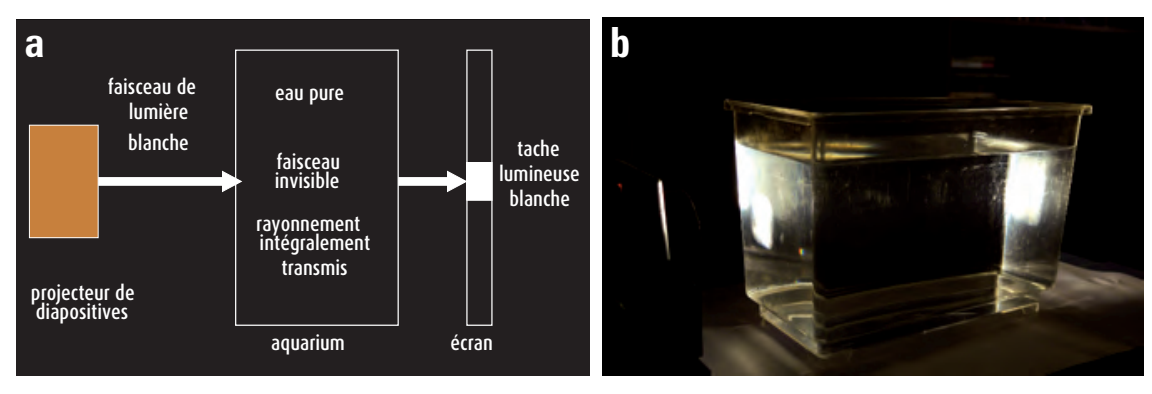

Figure 1. a) Schéma du dispositif expérimental de la première expérience. b) Photographie du bac traversé par le faisceau lumineux.

\section{Sans atmosphère pour diffuser la lumière, le ciel serait noir}

\section{Première expérience}

Remplir un aquarium avec de l'eau aussi propre que possible. Il est conseillé de laisser l'eau reposer suffisamment longtemps pour que toutes les micro-bulles aient pu remonter à la surface et de mettre un couvercle pour éviter que des poussières se déposent sur l'eau. Poser l'aquarium face au projecteur, le faisceau lumineux traversant l'aquarium. On ne voit pas le faisceau. Placer un écran blanc (ou une feuille blanche) au passage du faisceau sortant de l'aquarium : la tache lumineuse sur l'écran est blanche (figure 1a). Si on place un écran noir sur un côté du récipient et que l'on regarde par l'autre côté du récipient, l'écran reste noir (figure 1b). 


\section{Absorption et diffusion}

Lors de son trajet dans l'atmosphère, le rayonnement incident émis par le Soleil peut être absorbé ou diffusé par les composants atmosphériques (molécules et particules) (figure E1).

Dans le cas de l'absorption, le rayonnement cède partiellement ou totalement son énergie aux composants :

il y a donc atténuation du rayonnement dans la direction de propagation. L'intensité de cette absorption dépend de la longueur du trajet dans le milieu (plus ce trajet est long, plus l'absorption sera forte), de la densité et de la nature des composants du milieu, et de la longueur d'onde du rayonnement.

La diffusion est, quant à elle, la redistribution spatiale de l'énergie d'une onde lumineuse incidente lorsqu'elle rencontre une hétérogénéité (un diffuseur) dans le milieu de propagation. Elle dépend du trajet optique dans le milieu de propagation, de la longueur d'onde du rayonnement, ainsi que de la nature, la taille, la forme et le nombre des diffuseurs (molécules, aérosols, gouttelettes).

Dans le spectre visible, les particules atmosphériques (aérosols et gouttelettes) sont principalement diffusantes; les molécules sont peu absorbantes mais très diffusantes.

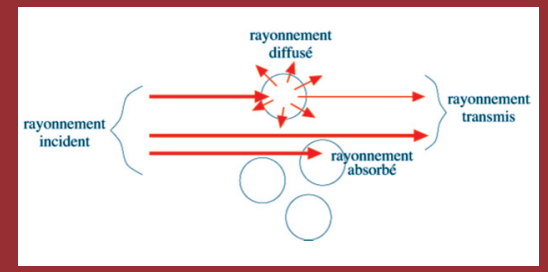

Figure E1. Schéma très simplifié des principaux mécanismes que peut subir le rayonnement lumineux en rencontrant des molécules ou particules : transmission, absorption, diffusion.

\section{Un peu d'histoire}

Léonard de Vinci (1452-1519) s'était rendu compte, lors de ses études pour les peintures de paysages, que les objets très distants apparaissaient d'une couleur plus bleutée, que les ciel apparaissait plus blanc lorsqu'il était vu près de l'horizon et que, lorsque l'on faisait une ascension dans les Alpes, le ciel apparaissait plus bleu et les rayons du Soleil plus lumineux. Il avait attribué cela à la diminution de la couche d'atmosphère et en avait conclu qu'au-dessus de celle-ci le ciel devait apparaître noir. II pensait que la couleur bleue était due à la vapeur d'eau présente dans I'atmosphère (Lilienfeld, 2004). Quelque 200 ans plus tard, Isaac Newton (1642-1727) s'intéressa aussi à cette question, mais il ne fournit pas de meilleure explication.
Horace Benedict de Saussure (1740-1799), géologue, météorologue, physicien et botaniste de Genève, réalisa de nombreuses expériences au sommet du mont Blanc, qu'il considérait être un laboratoire de choix pour l'étude des phénomènes météorologiques et atmosphériques. II suscita la première ascension du mont Blanc en 1786 et fera lui-même cette ascension en 1787

Saussure mit au point un appareil pour mesurer la quantité de bleu du ciel à différentes altitudes. En effet, il souhaitait déterminer la quantité de vapeur qui diminue la transparence de l'air et donne en conséquence sa couleur bleue au ciel

" La couleur du ciel peut être considérée comme la mesure de la quantité des vapeurs opaques, ou des exhalaisons qui sont suspendues dans l'air. Car il est bien prouvé que le ciel paraîtroit absolument noir, si l'air étoit parfaitement transparent, sans couleurs. » (Cordier).

En 1859, Tyndall, aussi amateur de montagne, est le premier à réaliser une expérience qu'il appela " le ciel dans une boîte ». II utilisa un cylindre en verre de $90 \mathrm{~cm}$ de long et de $7 \mathrm{~cm}$ de diamètre, éclairé à une extrémité par un faisceau de lumière blanche, dans lequel il introduisit différents gaz (Tyndall, 1869). En insérant de la fumée, il remarqua une variation de couleur du faisceau passant du bleu au rouge à l'autre bout. C'est ce qu'on appelle l'effet Tyndall. En faisant l'analogie avec I'atmosphère, Tyndall émit I'hypothèse que les particules de l'air diffusaient prioritairement les courtes longueurs d'onde, donc le bleu.

Ce phénomène est plus communément connu sous le nom de " diffusion de Rayleigh », car il a ensuite été étudié mathématiquement par John William Strutt (connu sous son titre de Lord Rayleigh, 1873) ((Bohren et Fraser, 1985 ; Strutt, 1871). Cependant, Tyndall et Rayleigh pensaient que la lumière était diffusée par de très fines gouttelettes d'eau et des petites poussières.

Par la suite, Smoluchowski puis Einstein (en 1911) montrèrent que ce sont les molécules de gaz (azote et oxygène) qui diffusent la lumière bleue.

Les diffusions de Rayleigh, de Mie et de l'optique géométrique

Pour calculer l'interaction entre ondes électromagnétiques et particules, il faut résoudre les équations relatives aux ondes électromagnétiques (équations de Maxwell) dans les particules et dans le milieu qui les entoure. Pour les particules de forme quelconque, il faut résoudre numériquement ces équations en discrétisant finement l'espace, ce qui est très difficile à faire et très coûteux à calculer. Pour les particules de formes sphérique, des développements analytiques permettent d'éviter la discrétisation spatiale. Il s'agit de la " théorie de Mie » (du nom du physicien allemand Gustav Mie, 1908) qui s'exprime sous la forme d'équations plus simples que celles générales mais qui restent encore très difficiles à résoudre. Si les particules sphériques sont de taille très inférieure à la longueur d'onde, ces équations se simplifient et tendent vers celles de la " diffusion de Rayleigh ». Dans le visible et I'infrarouge, la diffusion de Rayleigh concerne les molécules et particules de taille nanométrique. La puissance diffusée est inversement proportionnelle à la puissance 4 de la longueur d'onde ; elle est donc plus importante pour les courtes longueurs d'onde. À titre d'exemple, pour la lumière blanche incidente du Soleil, la diffusion atmosphérique est environ cinq fois plus élevée dans le bleu $(\lambda \sim 480 \mathrm{~nm})$ que dans le rouge $(\lambda \sim 700 \mathrm{~nm})$.

Si les particules sont de grande taille par rapport à la longueur d'onde, alors les équations de Mie se simplifient également et tendent vers celles de l'optique géométrique. La puissance du rayonnement diffusé dépend de la variation de l'indice de réfraction avec la longueur d'onde, dépendance qui est en général assez faible. Si les particules ont une taille proche de la longueur d'onde, il faut alors résoudre les équations de Mie. La puissance du rayonnement diffusé dépend de la longueur d'onde mais beaucoup moins que la diffusion de Rayleigh.

Par conséquent, pour le rayonnement visible et dans l'exemple de molécules de gaz ou des macromolécules du lait, le régime de diffusion est celui de Rayleigh avec une très grande dépendance à la longueur d'onde. Dans l'exemple de gouttelettes d'eau d'un nuage (de diamètre compris entre 5 et $40 \mu \mathrm{m}$ ), ou de grains de talc dans l'eau, le régime de diffusion est celui de Mie ou celui de l'optique géométrique et il est assez uniforme sur toutes les couleurs du spectre.

Enfin, l'intensité de la lumière diffusée augmente avec la différence entre l'indice de réfraction du diffuseur et celui du milieu environnant. À titre d'exemple, les peintures blanches modernes contiennent des particules minérales choisies pour leur indice de réfraction $m$ très élevé, telle que le cinabre $(m>3)$, comparé à l'indice du liant, par exemple I'huile $(m \approx 1,5)$. Ces diffuseurs sont donc le siège $d^{\prime} u n e$ diffusion importante et produisent une couleur blanche et éclatante. Par contre, les nuages sont d'un blanc moins intense, car les gouttelettes d'eau qui diffusent le rayonnement solaire incident ont un indice qui est seulement légèrement plus élevé $(m \approx 1,3)$ que celui de l'air environnant $(m=1)$. 


\section{Interprétation}

L'eau pure ne diffuse pas la lumière, pour des raisons physiques dont l'explication dépasse le cadre de cet article. En conséquence, on ne voit pas le faisceau si l'œil n'est pas dans son axe. Pour voir un objet (ou un milieu), il faut que celui-ci émette ou diffuse de la lumière dans la direction de l'œil. On ne voit pas le faisceau lumineux du projecteur dans l'eau, comme on ne voit pas les faisceaux de lumière solaire dans l'espace. On peut faire une expérience similaire avec un pointeur laser, dont on ne voit pas le trajet du faisceau, sauf à le saupoudrer d'un peu de craie.

L'eau illustre ce qui se passerait sur une planète sans atmosphère. De même qu'un écran noir placé le long du récipient apparaît noir quand on le regarde à travers l'eau, le ciel c'est-à-dire ce que l'on voit depuis la Terre - apparaîtrait noir s'il n'y avait pas d'atmosphère : c'est elle qui diffuse et rend visible la lumière. De même, l'espace apparaît noir dès que l'on sort de l'atmosphère terrestre (figure 2).

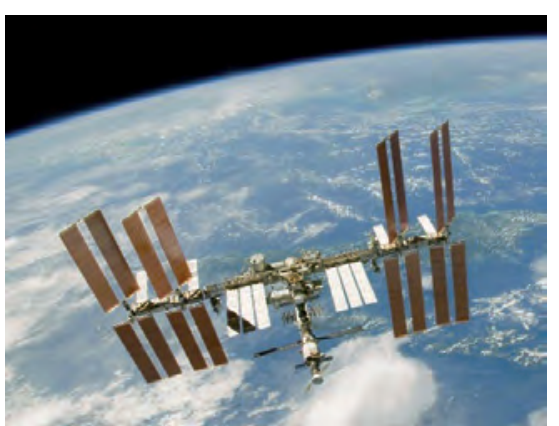

Figure 2. Photographie de la Station spatiale internationale prise depuis la navette spatiale Endeavour le 20 février 2010. L'espace apparaît noir, car la lumière n'y est pas diffusée.

\section{Pourquoi les nuages sont-ils blancs ou gris?}

\section{Deuxième expérience}

Dans un autre aquarium rempli d'eau, introduire de la poudre de talc. Le talc se mélangeant difficilement à l'eau, il est conseillé de bien diluer le talc au préalable dans un petit récipient d'eau, puis de verser une cuillère à café du mélange dans l'aquarium. Bien mélanger. Poser l'aquarium face à l'éclairage, un écran blanc de l'autre côté, le faisceau lumineux traversant l'aquarium dans le sens de la largeur (figure $3 \mathrm{a}$ ).
On observe que le faisceau est immédiatement visible, que la lumière diffusée est blanche (figure $3 b$ ) et que la tache lumineuse sur l'écran que l'on peut disposer au bout de l'aquarium est blanchâtre ou d'un gris très clair. Si on augmente la quantité de talc ou que l'on tourne l'aquarium de façon à ce qu'il soit éclairé dans le sens de la longueur, la lumière diffusée reste blanche, mais la tache sur l'écran prend une couleur plus grisée. Si on place un écran noir sur le côté, il n'apparaît plus noir mais prend une couleur gris foncé.

\section{Interprétation}

Le talc est formé de particules relativement grosses par rapport à la longueur d'onde de la lumière. Elles diffusent la lumière selon la diffusion dite de Mie ou selon les lois de l'optique géométrique (encadré 1). Ce type de diffusion sépare peu les couleurs. Autrement dit, toutes les longueurs d'onde sont diffusées presque de la même façon. Les faisceaux de lumière diffusés et transmis sont tous blancs (figure 3).
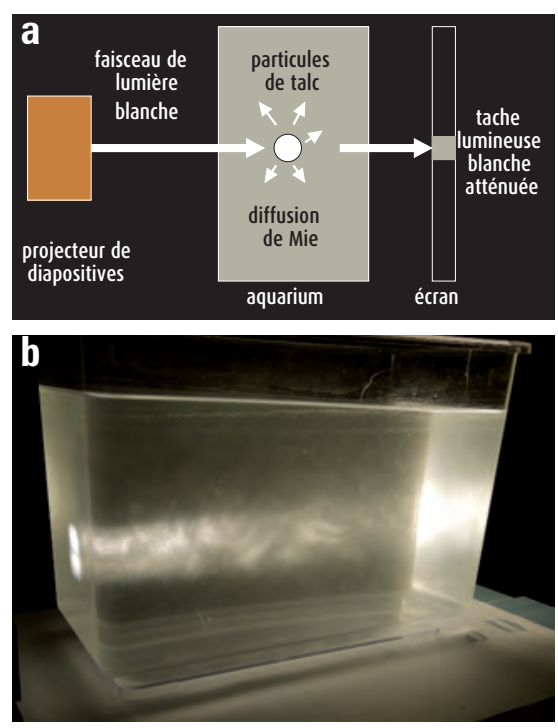

Figure 3. a) Schéma du dispositif expérimental de la deuxième expérience. b) Photographie du bac traversé par le faisceau lumineux.

Dans l'atmosphère, les nuages sont formés de gouttelettes ou cristaux de glace dont la taille est plus grande d'environ un facteur 10 - que la longueur d'onde de la lumière. La lumière est alors diffusée selon la diffusion de Mie ou de l'optique géométrique. C'est la raison pour laquelle les nuages apparaissent blancs. Lorsque les nuages sont épais, comme dans le cas des nuages d'orage, peu de lumière parvient à les traverser.
On les voit alors gris (figure 4) voire presque noirs s'ils sont très épais (cumulonimbus).

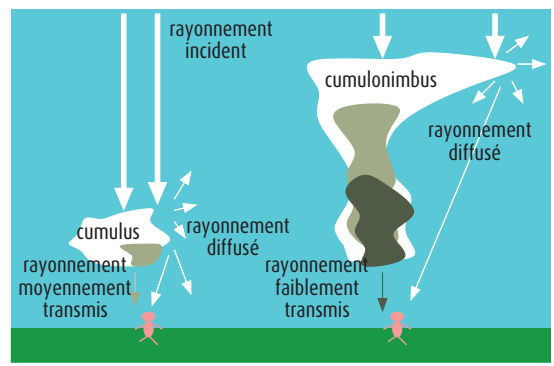

Figure 4. Schéma expliquant pourquoi les nuages sont blancs ou gris pour un observateur au sol. Si l'observateur reçoit surtout la lumière diffusée, blanche, il voit le nuage blanc. Si l'observateur ne reçoit qu'une petite partie de la lumière incidente, à cause de la réflexion, diffusion ou absorption, il voit le nuage ou les parties de nuage grises, d'autant plus foncées qu'il reçoit moins de lumière incidente.

\section{Pourquoi le ciel est-il bleu?}

\section{Troisième expérience}

Remplir d'eau le troisième aquarium. $\mathrm{Au}$ lieu du talc, ajouter quelques gouttes de lait puis mélanger. Cette expérience est très sensible à la quantité de lait introduite : quelques pincées de lait en poudre ou une cuiller à café de lait liquide devraient suffire (figure 5).
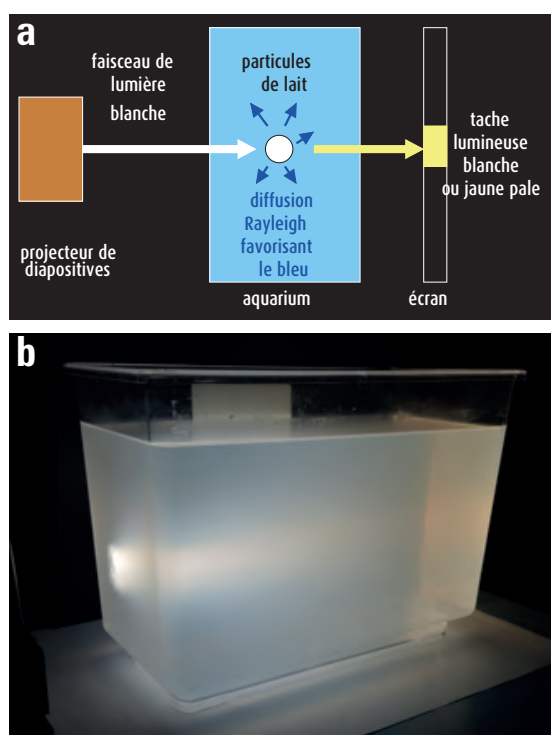

Figure 5. a) Schéma du dispositif expérimental de la troisième expérience. b) Photographie du bac traversé par le faisceau lumineux. On peut voir la coloration bleutée de l'eau dans la moitié du récipient près de la face d'entrée du faisceau. L'eau devient jaune dans la partie droite du récipient. 
Poser l'aquarium de façon à ce que le faisceau lumineux le traverse dans le sens de la largeur. On constate que le faisceau est bien visible et que l'eau prend une coloration - légèrement bleutée, surtout vers la face d'entrée du faisceau lumineux. Le faisceau est blanc près de l'entrée dans l'eau, puis devient plus jaune au bout d'une dizaine de centimètres de parcours. De même, l'eau prend une couleur jaune d'autant plus marquée que le faisceau a traversé une plus grande distance. La tache lumineuse sur l'écran blanc varie de blanche à jaune clair suivant la quantité de lait (figure 5b). L'écran noir placé sur

\section{Pourquoi l'eau contenant quelques gouttes de lait est-elle bleutée?}

La lumière incidente atteignant le liquide est en partie diffusée et en partie transmise par les molécules (l'absorption peut être négligée dans ces expériences).

Nous avons vu que les molécules du lait, qui sont de petite taille, diffusent davantage le rayonnement de petite longueur d'onde que celui de grande longueur d'onde : autrement dit, le bleu est plus diffusé que le rouge. C'est pour cela que lorsque le lait est très dilué, la solution obtenue est bleutée.

Lorsque le lait est moins dilué, le rayonnement bleu est toujours entièrement diffusé. Cependant, en raison du beaucoup plus grand nombre de molécules de lait, les rayonnements vert, jaune, orange et rouge sont également diffusés. Il n'y a plus de lumière qui arrive à traverser le récipient sans diffusion comme c'était le cas lorsque le lait était dilué. Tout le rayonnement est donc diffusé quelle que soit sa longueur d'onde et le lait apparaîtra blanc s'il est éclairé par une lumière blanche (figure E2).

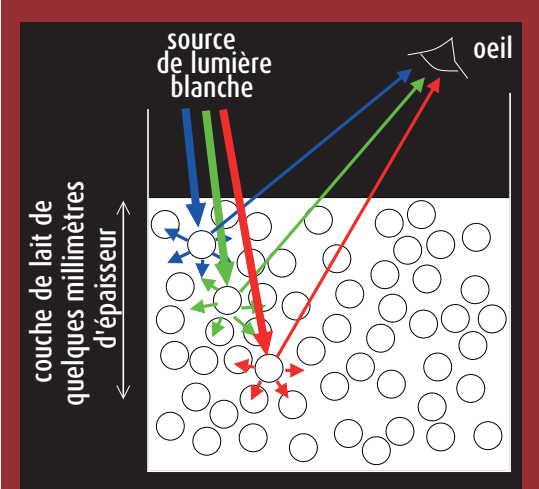

Figure E2. Schéma montrant pourquoi l'œil humain voit le verre de lait blanc, alors que les particules de lait diffusent préférentiellement la lumière bleue. le côté de l'aquarium, qui est vu à travers l'eau diffusant le bleu, n'apparaît plus aussi sombre, mais on voit distinctement le bleu diffusé par l'eau.

Pour que le phénomène soit bien visible, il est nécessaire d'utiliser un projecteur de diapositives, car sa lampe halogène produit une lumière qui contient une composante bleue suffisante. Pour obtenir un faisceau suffisamment fin, il est possible d'insérer une diapositive percée d'une fente. Par contre, un éclairage avec une lampe de poche standard ne fonctionnera pas, sa lumière ne contenant presque pas de bleu. Les lampes LED ou d'un vidéo-projecteur ne conviendront pas non plus car leur spectre d'émission est discret.

\section{Interprétation}

Les particules de lait, bien plus fines que les particules de talc, obéissent à la diffusion de Rayleigh qui dépend de la longueur d'onde. Elles diffusent donc beaucoup plus efficacement le rayonnement de courte longueur d'onde, c'est-à-dire le bleu (encadré 1). C'est la raison pour laquelle l'aquarium prend une coloration bleutée. Pour les curieux, l'encadré 2 explique pourquoi un verre de lait apparaît blanc, bien que le lait diffuse prioritairement le bleu.

En quoi cela est-il analogue avec ce qu'il se passe dans l'atmosphère ? Le Soleil éclaire la Terre avec de la lumière blanche (encadré 3a). L'atmosphère est constituée de molécules de gaz, $78 \%$ de diazote $\left(\mathrm{N}_{2}\right)$ et $21 \%$ de dioxygène $\left(\mathrm{O}_{2}\right)$. Comme les fines particules de lait, ces molécules diffusent préférentiellement la lumière bleue. C'est la raison principale pour laquelle le ciel prend une coloration bleue.

Cependant, la lumière solaire contient aussi du violet et de l'indigo. Ces couleurs ne sont pas perçues par un observateur au sol pour les deux raisons suivantes. La première est que le spectre solaire contient beaucoup moins de violet et d'indigo que de bleu (figure E3a); ces deux couleurs, de longueurs d'onde plus courtes que le bleu, sont déjà entièrement diffusées lorsque la lumière solaire a traversé l'atmosphère et arrive au sol. Pour un observateur situé à haute altitude, le ciel contient plus d'indigo que lorsqu'il est au niveau de la mer. La seconde raison est que, pour la très grande majorité des personnes, l'œil humain est moins sensible au violet et à l'indigo qu'au bleu (figure E3b).
La diffusion de la lumière du Soleil par les molécules de l'atmosphère est aussi la principale raison pour laquelle la Terre apparaît bleue quand elle est vue de l'espace (figure 2).

Quand on regarde le ciel près de l'horizon, le ciel est d'une couleur bleue beaucoup plus pâle. C'est parce que la concentration d'aérosols (poussières, pollens, particules de sel marin...) est plus élevée dans les basses couches de l'atmosphère, et que ces aérosols ont un diamètre suffisamment gros, surtout si l'humidité est élevée,

\section{La lumière}

La lumière est un rayonnement électromagnétique constitué d'une superposition d'ondes. L'œil humain perçoit les longueurs d'onde comprises entre 380 et $750 \mathrm{~nm}$ (figure E3a). Chaque longueur d'onde est interprétée par le cerveau comme une couleur. Par exemple, des rayonnements de longueur d'onde de 380, 600 et $750 \mathrm{~nm}$ sont perçus respectivement comme du bleu, du jaune et du rouge.

Le Soleil rayonne dans une large gamme de longueurs d'onde : c'est une source dite polychromatique. Son irradiance est maximale dans la gamme de longueurs d'onde visibles. L'œil humain voit une lumière blanche, mais celle-ci est en fait constituée d'une superposition de l'ensemble des couleurs de l'arc-en-ciel. Cela a été démontré en 1672 par Isaac Newton qui avait mis un prisme sur le trajet de la lumière et avait constaté que le faisceau lumineux se décomposait du violet au rouge en passant par les couleurs indigo, bleue, verte, jaune et orange. Parmi ces couleurs, l'œil humain est sensible préférentiellement au bleu, puis au rouge et au vert (figure E3b).

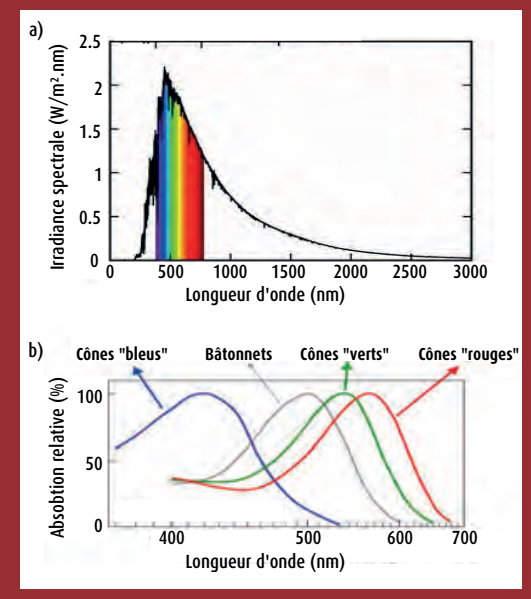

Figure E3. a) Spectre de la lumière solaire en fonction de la longueur d'onde. La lumière visible correspond à des longueurs d'onde comprises entre 380 et $750 \mathrm{~nm}$. b) Spectre de réponse des photorécepteurs de l'œil humain, d'après Bowmaker et Dartnall (1980). 
pour que la diffusion des courtes longueurs d'onde (le bleu) soit nettement moins importante que lorsque l'air est pur. Pour ces aérosols, toutes les longueurs d'onde sont diffusées de la même façon (diffusion de Mie). Cette diffusion rajoute du «blanc» au bleu du ciel et le rend donc plus pâle, ce qui se voit bien lorsqu'on regarde près de l'horizon.

\section{Pourquoi le ciel est-il rouge au lever ou au coucher de soleil ?}

\section{Quatrième expérience}

Placer l'aquarium contenant le lait de façon à ce que le faisceau le traverse dans le sens de la longueur (figure 6). On constate que le faisceau blanc devient progressivement de plus en plus jaune, voire orange, au fur et à mesure qu'il pénètre dans l'aquarium. À la sortie de l'aquarium, la lumière est orange.
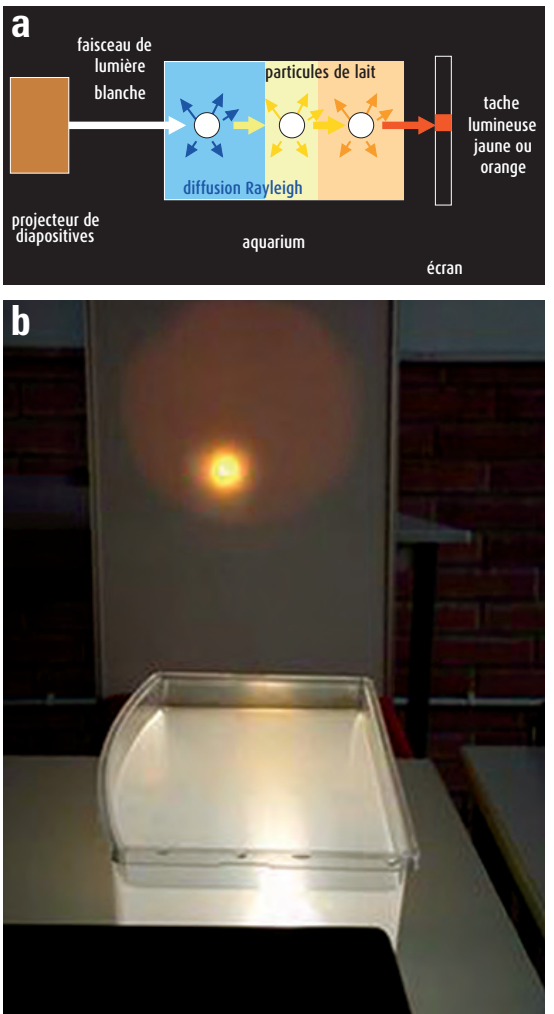

Figure 6. a) Schéma du dispositif expérimental de la quatrième expérience. Le faisceau traversant le bac permet d'illustrer que le ciel devient orangé au lever ou au coucher de soleil. b) Photographie du bac traversé par le faisceau lumineux qui devient orangé. On voit la tache orangée sur l'écran.

\section{Interprétation}

La lumière est d'autant plus diffusée que sa longueur d'onde est petite, c'est-à-dire que la couleur est proche du bleu ou du violet. Inversement, la lumière est d'autant moins diffusée, et donc d'autant plus transmise, que sa longueur d'onde est grande, c'est-à-dire qu'elle tend vers le rouge. Ainsi, le faisceau qui traverse l'aquarium dans toute sa longueur prend une couleur orange ou rouge (figure 6).

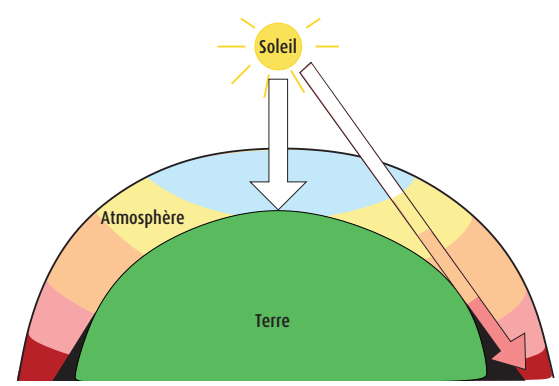

Figure 7. Schéma montrant pourquoi le ciel est bleu à midi et devient orangé au lever ou au coucher de soleil.

La part de lumière diffusée augmente avec la longueur de milieu diffusant traversée. À midi, les rayons du soleil traversent une épaisseur d'atmosphère minimale. Une partie du rayonnement bleu est diffusée, donnant la couleur bleue du ciel, mais suffisamment peu pour que la lumière du soleil reste blanche. Il est fortement déconseillé de regarder le soleil en face pour vérifier ! $\mathrm{Au}$ lever ou au coucher du soleil, au contraire, les rayons du soleil traversent une épaisseur d'atmosphère bien plus importante. Les rayonnements de faible longueur d'onde sont complètement diffusés avant d'arriver à l'œil de l'observateur au sol, d'où la coloration orangée, voire rouge suivant les conditions (pollution, aérosols, etc.), qu'il perçoit (figure 7).

Notons que si l'atmosphère était plus épaisse, le ciel n'apparaîtrait pas bleu pendant la journée, mais prendrait une coloration jaune ou orangée.

\section{Coucher de soleil sur Mars}

La diffusion de Rayleigh (du bleu) par les molécules de l'atmosphère martienne est beaucoup moins forte que sur Terre, car l'atmosphère y est environ 200 fois plus mince. Toutefois, comme sur Terre, l'atmosphère martienne joue un rôle déterminant dans la couleur du ciel. Mars est recouverte d'une fine poussière contenant de l'oxyde de fer, souvent propulsée en grandes quantités dans l'atmosphère par de fréquentes tempêtes. Ces poussières, qui donnent au sol martien un aspect rougeâtre, diffusent la lumière rouge et absorbent la lumière bleue émises par le Soleil (Ehlers et al., 2014). C'est d'ailleurs ces mêmes poussières qui donnent au ciel de Mars une teinte brune les jours d'été ensoleillés.

Par analogie avec la quatrième expérience, lorsque le Soleil est proche de l'horizon, les longueurs d'onde rouge - ici, les plus diffusées sont largement absentes du spectre lumineux. Toutefois, dans le cas présent et à la différence de la quatrième expérience, les longueurs d'onde bleues sont également largement absorbées par les poussières martiennes. Au final, la diffusion du rouge étant légèrement plus efficace que l'absorption du bleu, la résultante des deux effets donne au disque solaire observé par les robots martiens une couleur blanche, légèrement teintée de bleu (figure 8).

Enfin, il faut invoquer un élément supplémentaire pour expliquer la présence de la lueur bleue intense tout autour du disque solaire : la direction de la diffusion. Les grains de poussières martiens diffusent certes majoritairement la lumière rouge, mais ils diffusent la lumière bleue bien plus efficacement vers l'avant. Voilà pourquoi les robots martiens peuvent observer un magnifique ciel bleu à proximité du soleil couchant et levant.

\section{Conclusion}

Cette série d'expériences permet d'aborder simplement et de façon ludique certaines des raisons pour lesquelles le ciel prend différentes couleurs. Elles peuvent paraître simples, mais elles mettent en jeu des processus sous-jacents très complexes. La couleur bleue du ciel constituait une « énigme » pour les scientifiques qui se sont intéressés à cette question lors des derniers siècles.

Aujourd'hui, la composition de l'atmosphère ainsi que son évolution sont étudiées en particulier via les observations satellitaires, grâce aux phénomènes physiques d'absorption et de diffusion décrits dans cet article. 


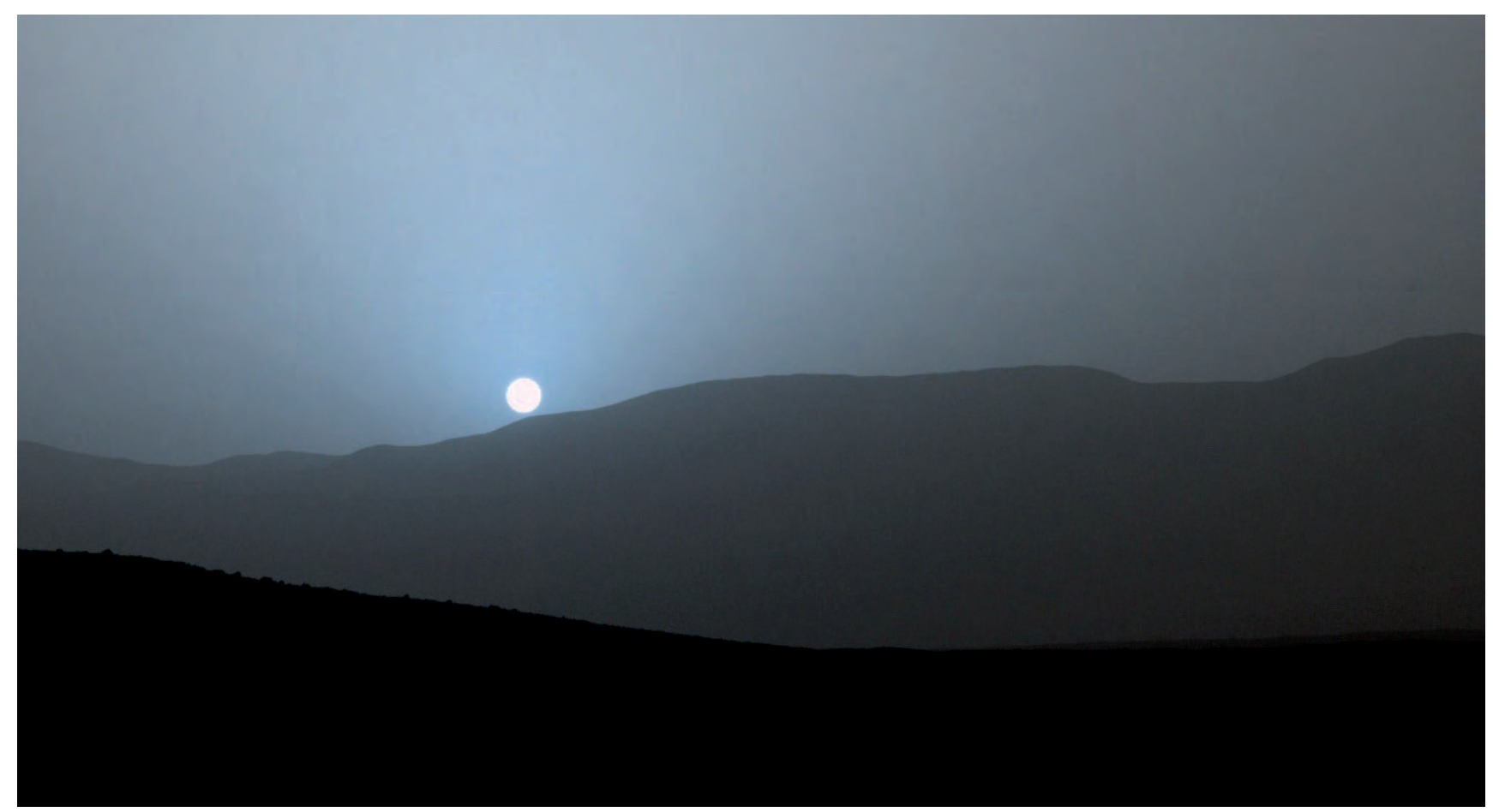

Figure 8. Photographie d’un crépuscule sur Mars, prise par le rover Curiosity de la Nasa et sa caméra Mastcam. La sensibilité spectrale de la caméra, très proche de celle d'un œil humain, sous-estime légèrement la couleur bleue. (C) Nasa.

Ces mêmes phénomènes physiques sont utilisés pour tenter de déterminer la composition de l'atmosphère des exoplanètes, telles que HD 189733B, une planète gazeuse géante qui serait de couleur bleue, peut-être en raison de nuages de haute altitude composés de minuscules gouttes de verre (Pont et
Evans, 2014). Les scientifiques observent ces couleurs à l'aide de puissants télescopes pour tenter d'interpréter la nature des atmosphères des planètes du Système solaire et même au-delà. Les planètes extrasolaires, composées pour certaines d'une atmosphère d'hydrogène, pour d'autres de nuages de zinc ou encore de verre, exhibent une variété sans précédent de couleurs qu'il nous appartient d'explorer... Beaucoup de choses restent donc encore à découvrir grâce aux « couleurs du ciel » des planètes.

\section{Bibliographie}

Bohren C.F., Fraser A.B., 1985. Colors of the sky. The Physics Teacher, 23, 267-272. doi: 10.1119/1.2341808

Bowmaker J.K., Dartnall H., 1980. Visual pigments of rods and cones in a human retina. J. Physiol., 298, 501-511.

Cordier S., La mesure du bleu du ciel. Disponible à l'adresse : www.fondation-lamap.org/sites/default/files/upload/media/minisites/projet_europe/PDF/sausHistfr.pdf Dufresne J.-L., 2002. La Physique de l'atmosphère. Graines de Sciences 4, 59-94, Éditions Le Pommier, Paris.

Ehlers K., Chakrabarty R., Moosmüller H., 2014. Blue moons and Martian sunsets. Appl. Opt., 53, 1808-1819. doi: 10.1364/A0.53.001808

Lilienfeld P., 2004. A blue sky history. Opt. Photonics News, 15, 32-39. doi: 10.1364/OPN.15.6.000032

Pont F., Evans T., 2014. Les couleurs des exoplanètes. Pour la Science, 438, 20-27.

Strutt J. (Lord Rayleigh), 1871. On the light from the sky, its polarization and colour. Philos. Mag., Series 4, 41, 107-120, $274-279$.

Tyndall J., 1869. On the blue color of the sky, the polarization of skylight, and on the polarization of light by cloudy matter generally. Proc. R. Soc. London, 17, 223-233. 